\title{
New Model and Sliding mode control of a three phase grid connected photovoltaic system without DC-DC Converter
}

\author{
Hicham Bahri ${ }^{1}$, Mohamed Aboulfatah ${ }^{1}$, M'hamed Guisser ${ }^{1}$, \\ Elhasan Abdelmounim ${ }^{1}$, Mohammed El Malah ${ }^{1}$, Adekanle simon ${ }^{1}$ \\ ${ }^{1}$ Department of Physics, Laboratory ASTI, FST-Settat, University \\ Hassan I, Settat, Morocco (e-mail: hbahri.inf@gmail.com).
}

\begin{abstract}
This paper presents a new model and an advanced control strategy of a three phase grid connected photovoltaic system without DC-DC converter. The novel model is characterized by decoupled inputs and high relative degree of the outputs. A sliding mode control without chattering phenomena based on this new model is applied in order to control the system to track the maximum power point produced by the photovoltaic generator PVG, convert all this power to active power and compensate the harmonic components and reactive power caused by nonlinear load if it is connected to the PV system. This controller is able to realize all these objectives with a high dynamic performance in presence of changing atmospheric conditions, system's disturbance and with any kind of load or without load. Simulation results demonstrate efficient performance and robustness of the proposed strategy.
\end{abstract}

Keywords: Inverter, Maximum Power Point Trackers, Unity power factor, Photovoltaic Generator, Power Conversion Harmonics, Pulse Width Modulation Converters ,Reactive power compensation, Nonlinear load.

\section{Introduction}

Recently, due to environmental pollution and depletion of fossil fuels, renewable energy sources have gained more attention of researchers and investors, and in particular, research on photovoltaic systems is constantly being conducted [1]. Especially, about the photovoltaic system connected to the utility grid, that produces electricity at a site close to customers or that is tied to an electric distribution system [2], this system can be realized by using a DC-DC converter and inverter (Tow-Stage topology), or just an inverter (Single-stage topology). The second topology is more economical owing to the elimination of DC-DC converter. This also decreases the number of the sensors needed by the controller and thereby reduces measurement errors. This topology has gained attention, especially in low voltage application [3]. This work is devoted to optimize the operation of a three phase grid single stage connected photovoltaic system by controlling the voltage source inverter VSI in order to realize the following objectives:

- Tracking maximum active power MPPT;

- Generate maximum available active power to feed a load and supply the grid;

- Compensate harmonic components and reactive power caused by nonlinear load.

Many control strategies have been designed to achieve these objectives [4]-[8], but they aren't able to control the system to track precisely the maximum power produced by the 
photovoltaic generator PVG or they are not good compensators of current's harmonics components caused by nonlinear load.

This paper presents, for the first time, a new model of the studied system. This model decouples the control input of the inverter and increases the relative degree of the first output selected to be controlled in this work, which enhances the dynamic performance of the controller in the regulation of this output. After that, the paper proposes a new control strategy of the studied system based on the new model in dq0 axis applying a sliding mode technique [9], which is characterized by its efficient and robust dynamic performance. Furthermore, the reactive power needed or caused by nonlinear load is calculated by using a new simplify mathematic expression in dq0 axis. As results of this work, the elaborated controller achieved all the objectives with a fast response time and very good precision in presence of changes in atmospheric conditions (irradiation and temperature) and system perturbation (parameters disturbance and harmonics pollution caused by nonlinear load). This later achieved performance is so attractive; it demonstrates the robustness of this strategy in front of systems disturbance compared to the other control strategies already deployed in the literature. The supplementary advantage of this controller is that it performs efficiently in presence of any kind of load or even without load in the case where the system will just supply the grid with the achievement of the unity power factor UPF.

Thereafter, the system description and modeling is presented in section 2 . The sliding mode control is developed in section 3. In section 4 the simulation results are illustrated using inductive load in order to demonstrate the performance of the controller. Finally, the paper presents a short conclusion..

\section{System description and modelling analysis}

The electrical schema of the studied system is shown in fig. 1. It consists of a PV Generator PVG coupled to a three phase grid and or without three phase load via a three phase Voltage source inverter VSI and a simple low pass filter $(\mathrm{L}, \mathrm{r})$. The capacitor $\mathrm{C}_{\mathrm{p}}$ is used to stabilize the input voltage of the inverter.

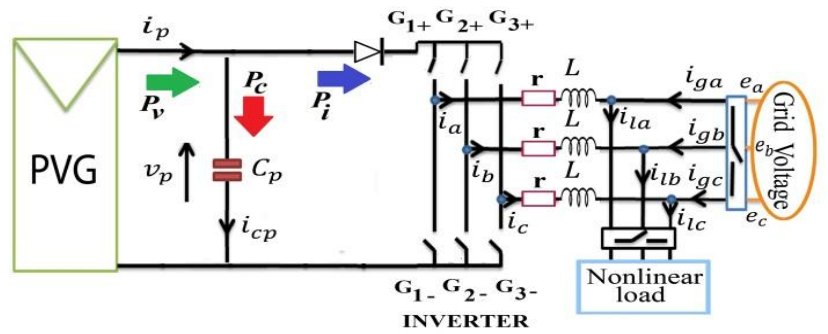

Fig. 1. Proposed topology for a PVG system connected to a three phase grid and any kind of load.

Where $\mathrm{i}_{\mathrm{p}}, \mathrm{v}_{\mathrm{p}}$ are the PVG current and voltage, $P_{v}$ is the PVG power, $P_{i}$ is the inverter active power. $i_{a}, i_{b}, i_{c}$ are the inverter currents. $e_{a}, e_{b}, e_{c}$ are the grid voltages, $C_{p}$ is the input capacitor of the inverter, $\mathrm{R}_{l}$ is the load resistance, $\mathrm{L}_{\mathrm{l}}$ is the load inductance, $\mathrm{i}_{\mathrm{ga}}, \mathrm{i}_{\mathrm{gb}}, \mathrm{i}_{\mathrm{gc}}$ are the grid currents, $i_{l a}, i_{l b}, i_{l c}$ are the load currents, $G_{1 \mp}, G_{2 \mp}, G_{3 \mp}$ are the switching signals of the inverter switchs.

The mathematical model of the global system is given by the following equations: 


$$
\left\{\begin{array}{c}
\frac{d v_{p}}{d t}=\frac{1}{C_{p}} \frac{P_{v}}{v_{p}}-\frac{1}{C_{p}} \frac{P_{i}}{v_{p}} \\
\frac{d i_{a}}{d t}=-\frac{r}{L} i_{a}-\frac{1}{L} e_{a}+\frac{1}{L} v_{a} \\
\frac{d i_{a}}{d t}=-\frac{r}{L} i_{b}-\frac{1}{L} e_{b}+\frac{1}{L} v_{b} \\
\frac{d i_{a}}{d t}=-\frac{r}{L} i_{c}-\frac{1}{L} e_{c}+\frac{1}{L} v_{c}
\end{array}\right.
$$

Wherve $\mathrm{v}_{\mathrm{a}}, \mathrm{v}_{\mathrm{b}}, \mathrm{v}_{\mathrm{c}}$ are the inverter voltages

The park's transformation matrix used in this paper is given by:

$$
\mathrm{M}_{\mathrm{abc}}^{\mathrm{dq} 0}=\frac{2}{3}\left(\begin{array}{ccc}
\sin (\theta) & \sin \left(\theta-\frac{2 \pi}{3}\right) & \sin \left(\theta-\frac{4 \pi}{3}\right) \\
\cos (\theta) & \cos \left(\theta-\frac{2 \pi}{3}\right) & \cos \left(\theta-\frac{4 \pi}{3}\right) \\
\frac{1}{2} & \frac{1}{2} & \frac{1}{2}
\end{array}\right)
$$

$\theta$ represents the phase angle of the grid calculated by PLL technique [10].

By using this matrix, the expressions of the active power and reactive power in $\mathrm{dq0}$ frame can be simplified by [11]:

$$
\begin{aligned}
P_{i} & =\frac{3}{2} E_{d} i_{d} \\
Q_{i} & =-\frac{3}{2} E_{d} i_{q}
\end{aligned}
$$

Using park's transformation, this model can be transformed into d-q axis and expressed in the following form:

Where:

$$
\left\{\begin{array}{c}
\frac{d v_{p}}{d t}=\frac{1}{C_{p}} \frac{P_{v}}{v_{p}}-\frac{1}{C_{p}} \frac{1}{v_{p}} \frac{3}{2} E_{d} I_{d} \\
\frac{d I_{d}}{d t}=-\frac{r}{L} I_{d}+w I_{q}-\frac{1}{L} E_{d}+\frac{1}{L} V_{d} \\
\frac{d I_{q}}{d t}=-\frac{r}{L} I_{q}-w I_{d}-\frac{1}{L} E_{q}+\frac{1}{L} V_{q}
\end{array}\right.
$$

$\left(\begin{array}{c}E_{d} \\ E_{q} \\ E_{0}\end{array}\right)=M_{a b c}^{d q 0}\left(\begin{array}{c}e_{a} \\ e_{b} \\ e_{c}\end{array}\right) ;\left(\begin{array}{c}I_{d} \\ I_{q} \\ I_{0}\end{array}\right)=M_{a b c}^{d q 0}\left(\begin{array}{c}i_{a} \\ i_{b} \\ i_{c}\end{array}\right) ;\left(\begin{array}{c}V_{d} \\ V_{q} \\ V_{0}\end{array}\right)=M_{a b c}^{d q 0}\left(\begin{array}{c}v_{a} \\ v_{b} \\ v_{c}\end{array}\right)$.

$\left(V_{d} V_{q}\right)$ is the input control vector considered in this work.

The advantage of this new model is that it decouples the inputs $V_{d}$ and $V_{q}$ such as $V_{d}$ control the direct component $I_{d}$ and $V_{q}$ control the quadratic component $I_{q}$ of the inverter output current, which enhances the stability of the global system. Moreover, the PVG output voltage $v_{p}$ isn't directly in function of the control inputs, which increases the relative degree of the first output chosen in this work. That enhances the stability degree of this output and eliminates the chattering phenomena of the sliding mode control law produced if the output has a relative degree less strictly than 2 . The chattering phenomena can saturate the inverter switches.

After having modelled the system, the next section presents the development of the control strategy.

\section{Control strategy}

The goal of this work is to elaborate a control strategy able to realize the following 
objectives:

$>$ Achieve the MPPT with excellent precision and very good response time in presence of irradiation and temperature changes.

$>$ Convert the maximum power generated by the PVG to active power.

$>$ Compensate the reactive power and harmonic components caused by load.

$>$ Guarantee the global asymptotic stability of the system

All these objectives must be realized with a high performance even in the presence of system perturbation. To achieve these objectives with the fixed performance, the sliding mode method is selected to develop the controller which is characterized by high dynamic performance and robustness.

The sliding mode control is described in the next sub sections.

\subsection{MPPT and active power control}

To achieve the MPPT and convert all the available PVG power to active power, the following output is selected to be controlled:

$$
Y_{1}=\frac{\delta P_{v}}{\delta v_{p}}=i_{p}+v_{p} \frac{\partial i_{p}}{\partial v_{p}}
$$

By controlling this output to converge to its reference $Y_{1 r e f}=0$, the system will track the MPP with good precision [12].

The sliding mode control law of the first output is developed as follows:

The relative degree of the output $Y_{1}$ is 2 . Therefore, the sliding mode surface must be defined as follows:

Where $\gamma_{1}$ is a positive constant.

$$
\sigma_{1}=\dot{\varepsilon}_{1}+\gamma_{1} \varepsilon_{1}
$$

$$
\varepsilon_{1}=Y_{1}-Y_{1 r e f}=i_{p}+v_{p} \frac{\partial i_{p}}{\partial v_{p}}
$$

And

$$
\dot{\varepsilon}_{1}=\left(2 \frac{\partial i_{p}}{\partial v_{p}}+v_{p} \frac{\partial^{2} i_{p}}{\partial v_{p}^{2}}\right) \dot{v}_{p}
$$

The first Lyapunov function can is defined by:

$$
V_{1}=\frac{1}{2} \sigma_{1}{ }^{2}
$$

So, its derivative is calculated as:

$$
\dot{V}_{1}=\sigma_{1} \dot{\sigma}_{1}
$$

$\dot{V}_{1}$ must be forced to be negative, in order to stabilize $\varepsilon_{1}$ to zero. For that $\dot{\sigma}_{1}$ can be selected as this form:

$$
\dot{\sigma_{1}}=-K_{1} \operatorname{sing}\left(\sigma_{1}\right)
$$

Where $K_{1}$ is a positive constant.

In other hand, $\dot{\sigma}_{1}$ can be expressed by using (7).

$$
\begin{gathered}
\dot{\sigma}_{1}=\left(2 \frac{\partial^{2} i_{p}}{\partial v_{p}{ }^{2}}+v_{p} \frac{\partial^{3} i_{p}}{\partial v_{p}{ }^{3}}\right) \dot{v}_{p}\left[\frac{P_{v}}{C_{p} v_{p}}-\frac{3 E_{d} I}{2 C_{p} v_{p}}\right]+\left(2 \frac{\partial i_{p}}{\partial v_{p}}+v_{p} \frac{\partial^{2} i_{p}}{\partial v_{p}^{2}}\right)\left[\frac{1}{C_{p}} \frac{\dot{P}_{v} v_{p}-P_{v} \dot{v}_{p}}{v_{p}{ }^{2}}\right]-\left(2 \frac{\partial i_{p}}{\partial v_{p}}+\right. \\
\left.v_{p} \frac{\partial^{2} i_{p}}{\partial v_{p}{ }^{2}}\right) \frac{3 E_{d}}{2 C_{p} v_{p}}\left[-\frac{r}{L} I_{d}+w I_{q}-\frac{1}{L} E_{d}+\frac{1}{L} V_{d}\right]+\left(2 \frac{\partial i_{p}}{\partial v_{p}}+v_{p} \frac{\partial^{2} i_{p}}{\partial v_{p}{ }^{2}}\right) \frac{3 E_{d^{I}}}{2 C_{p} v_{p}{ }^{2}} \dot{v}_{p}+\gamma_{1}\left(2 \frac{\partial i_{p}}{\partial v_{p}}+\right. \\
\left.v_{p} \frac{\partial^{2} i_{p}}{\partial v_{p}{ }^{2}}\right)\left[\frac{P_{v}}{C_{p} v_{p}}-\frac{3 E_{d} I_{d}}{2 C_{p} v_{p}}\right]
\end{gathered}
$$

By exploiting the equation (12) and (13) of $\dot{\sigma}_{1}, V_{d}$ can be calculated as follows: 


$$
\begin{gathered}
V_{d}=-\frac{2 C_{p} v_{p} L}{3 E_{d}\left(2 \frac{d i_{p}}{d v_{p}}+v_{p} \frac{\partial^{2} i_{p}}{\partial v_{p}^{2}}\right)}\left[-K_{1} \operatorname{sing}\left(\sigma_{1}\right)-\left(2 \frac{\partial^{2} i_{p}}{\partial v_{p}{ }^{2}}+v_{p} \frac{\partial^{3} i_{p}}{\partial v_{p}{ }^{3}}\right) \dot{v}_{p}\left[\frac{P_{v}}{C_{p} v_{p}}-\frac{3 E_{d} I_{d}}{2 C_{p} v_{p}}\right]-\left(2 \frac{\partial i_{p}}{\partial v_{p}}+\right.\right. \\
\left.v_{p} \frac{\partial^{2} i_{p}}{\partial v_{p}{ }^{2}}\right)\left[\frac{1}{C_{p}} \frac{\dot{P}_{v} v_{p}-P_{v} \dot{v}_{p}}{v_{p}^{2}}\right]-\left(2 \frac{\partial i_{p}}{\partial v_{p}}+v_{p} \frac{\partial^{2} i_{p}}{\partial v_{p}^{2}}\right) \frac{3 E_{d} I_{d}}{2 C_{p} v_{p}^{2}} \dot{v}_{p}-\gamma_{1}\left(2 \frac{\partial i_{p}}{\partial v_{p}}+v_{p} \frac{\partial^{2} i_{p}}{\partial v_{p}{ }^{2}}\right)\left[\frac{P_{v}}{C_{p} v_{p}}-\frac{3 E_{d} I_{d}}{2 C_{p} v_{p}}\right]+\left(2 \frac{\partial i_{p}}{\partial v_{p}}+\right. \\
\left.\left.v_{p} \frac{{\frac{\partial}{2} i_{p}}^{2}}{\partial v_{p}{ }^{2}}\right) \frac{3 E_{d}}{2 C_{p} v_{p}}\left[-\frac{r}{L} I_{d}+w I_{q}-\frac{1}{L} E_{d}\right]\right]
\end{gathered}
$$

This control law will force the Lyapunov function to be negative, which guarantees asymptotic convergence of the first output $Y_{1}$ to zero; achieving the MPPT with a high precision and fast response time. This performance can be regulated by using the controller parameters $K_{1}$ and $\gamma_{1}$. Furthermore, $v_{p}$ is stabilized at the optimum voltage corresponding to the MPP $P_{v \max }$. Therefore, the capacitor power $P_{C}$ is null. In fact,

$$
P_{C}=i_{c p} v_{p}=C_{p} v_{p} \frac{d v_{p}}{d t}
$$

which demonstrates that:

$$
P_{a}=P_{v \max }
$$

signifying that the inverter will convert all the available PVG power $P_{v \max }$ to active power.

\subsection{Reactive power control}

In order to guarantee the Unity Power Factor and the quality of the power in the level of the power flow between the system and the utility grid, the reactive power must be controlled to be null if there is a linear load or there isn't a load connected to the system ,or equal to reactive power needed or caused by a nonlinear load. This reactive power is calculated by using a new mathematical expression in $\mathrm{dq} 0$ axis as follows:

$$
Q_{L}=-\frac{3}{2} E_{d} I_{l q}
$$

Where:

$$
\left(\begin{array}{l}
I_{l d} \\
I_{l q} \\
I_{l 0}
\end{array}\right)=M_{a b c}^{d q 0}\left(\begin{array}{l}
i_{l a} \\
i_{l b} \\
i_{l c}
\end{array}\right)
$$

So, the inverter must generate a reactive power $Q_{i}$ such that:

$$
Q_{i}=Q_{L}
$$

The second output, which has been chosen to be controlled is:

$$
Y_{2}=I_{q}
$$

Using (4) the reference of this output is given by:

$$
Y_{2 r e f}=I_{\text {qref }}=-\frac{2}{3 E_{d}} Q_{L}
$$

The sliding mode control of the second output $Y_{2}$ is developed thereafter:

Firstly let's define the following tracking error:

$$
\varepsilon_{2}=Y_{2}-Y_{2 r e f}=I_{q}-I_{\text {qref }}
$$

The degree relative of $Y_{2}$ is 1

So, the sliding surface can be defined by:

$$
\sigma_{2}=\varepsilon_{2}
$$

The second Lyapunov function is expressed by: 


$$
V_{2}=\frac{1}{2} \sigma_{2}^{2}
$$

So, its derivative is:

$$
\dot{V}_{2}=\sigma_{2} \dot{\sigma}_{2}
$$

$\dot{V}_{2}$ must be negative under any condition. For that, let $\dot{\sigma}_{2}$ be:

$$
\dot{\sigma}_{2}=-K_{2} \operatorname{sat}\left(\sigma_{2}\right)
$$

Where $K_{2}$ is a positive constant and Sat is a function used to eliminate the chattering phenomena, because the second output has a degree relative equal to 1 . This function is defined by:

$$
\begin{aligned}
& \text { If absolute value of } \sigma_{2}<=\Delta \text { then } \\
& \qquad \operatorname{Sat}=\frac{\sigma_{2}}{\Delta} ; \\
& \text { Else } \operatorname{Sat}=\operatorname{sign}\left(\sigma_{2}\right) ; \\
& \text { End; }
\end{aligned}
$$

Where $\Delta$ is a setting parameter.

The derivative of $\sigma_{2}$ can be calculated by using (23) as follows:

$$
\dot{\sigma}_{2}=-w I_{d}-\frac{R}{L} I_{q}+\frac{1}{L} V_{q}-\frac{1}{L} E_{q}-\dot{I}_{q r e f}
$$

Therefore, the quadratic control law is given by:

$$
V_{q}=L\left[-k_{2} \operatorname{sat}\left(\sigma_{2}\right)+w I_{d}+\frac{r}{L} I_{q}+\frac{1}{L} E_{q}+\dot{I}_{\text {qref }}\right]
$$

This control input $V_{q}$ will drive $I_{q}$ to converge asymptotically to $I_{\text {qref }}$, which permits the compensation of reactive power and harmonic component caused by the load with a high performance.

To summarize, fig. 2 shows a block diagram of the PVG system and the proposed controller.

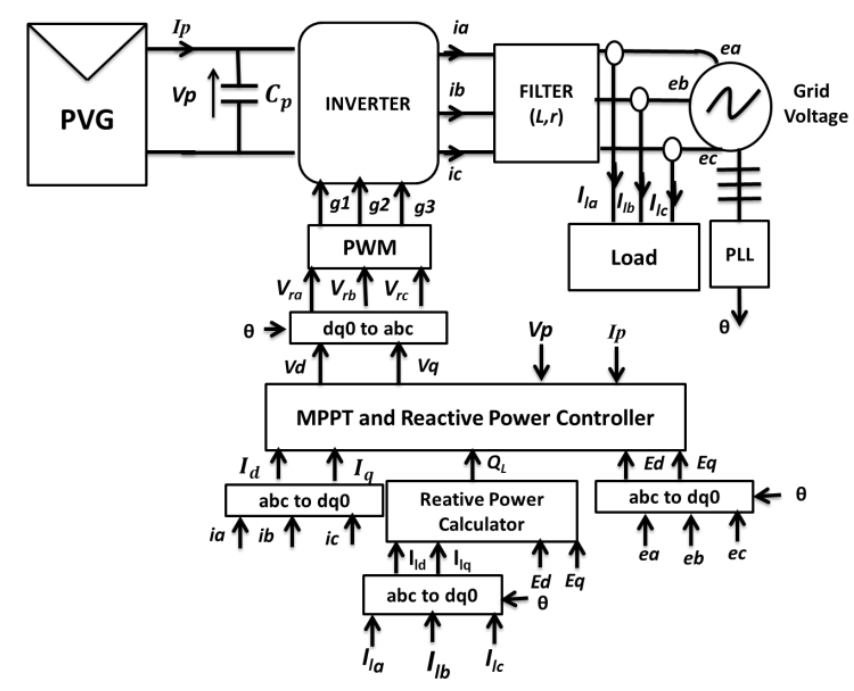

Fig. 2. Control block diagram of the PVG system and the proposed controller.

$\mathrm{v}_{\mathrm{ar}}, \mathrm{v}_{\mathrm{br}}, \mathrm{v}_{\mathrm{cr}}$ are the PWM voltage references and $\mathrm{g}_{1}, \mathrm{~g}_{2}, \mathrm{~g}_{3}$ are the switching signals of the inverter. The controller setting parameters are $\mathrm{K}_{1}, \gamma_{1}, \mathrm{~K}_{2}$ and $\Delta$. 
The control input $V_{d}$ and $V_{q}$ will force the derivative of the Lyapunov function $\dot{V}_{1}$ and $\dot{V}_{2}$ to be negative, which guarantees the asymptotic stability of the global system.

All this performance of the elaborated controller will be demonstrated in the next section by using the simulation results under MATLAB/SIMULINK.

\section{Simulation results}

In order to demonstrate the performance of the proposed controller, the paper presents the simulation results of the PVG system controlled by the sliding mode control simulated under Simulink/Matlab platform. The scenario used in this simulation is shown in Fig. 3. It presents the variation of climatic conditions (solar irradiation and temperature) at specific time. To demonstrate the robustness of the controller, the disturbance has been introduced at $12 \mathrm{~s}$ by changing the value of the input capacitor $C_{p}$ amount by $20 \%$ of its original values and at $15 \mathrm{~s}$, the load current will be polluted by about $10 \%$ of its fundamental value.

The PVG used in this work is formed by 50 Photovoltaic modules in parallel; every module consists of 20 Siemens SM55 panels in series. The SM55 generate a 55W at 1000 $\mathrm{W} / \mathrm{m}^{2}$ AND $25^{\circ} \mathrm{C}$. So, this PVG has a peak power of about $50 \mathrm{KW}$ at standard atmospheric conditions.

The nonlinear load used in this simulation is an inductive load; which could have been any kind of load. In fact, the controller is developed independently of the kind of load.

Table 1 presents the parameters of the system and the controller.

Table 1. Parameters of the System and the Controller

\begin{tabular}{cc}
\hline \hline System Parameters & Controller Parameters \\
\hline Input capacity: $\mathrm{C}_{\mathrm{P}}=4700 \mu \mathrm{F}$ & $\mathrm{K}_{1}=80000$ \\
& $\gamma_{1}=70$ \\
\hline Filter inductance: $L_{f}=3 \mathrm{mH}$ & $\mathrm{K}_{2}=110000$ \\
Filter resistance: $r=0.002 \Omega$ & $\Delta=30$ \\
\hline Load inductance: $L=20 \mathrm{mH}$ & PWM Frequency: $10 \mathrm{KHz}$ \\
Load resistance: $R=10 \Omega$ & \\
\hline Grid voltage: $380 \mathrm{~V}$ & \\
\hline Grid frequency: $50 \mathrm{~Hz}$ & \\
\hline \hline
\end{tabular}

Fig. 4 shows the control inputs $V_{d}$ and $V_{q}$. Its waveform doesn't contain distortion or fluctuation, which could have saturated the switching of the inverter by generating a high frequency PWM output control signal.

Fig. 5 shows the behavior of the first output $Y_{1}=\frac{\delta P_{v}}{\delta v_{p}}$. It converges to zero with a very fast response and very good precision under climatic condition changes and system disturbance. Moreover the MPPT is achieved with the same performance under any condition as shown in fig. 6, which permits to exploit the maximum power produced by the PVG. Furthermore, all the available PVG power is converted to active power as proved by fig. 7. In fact, the inverter active power tracks the PVG power efficiently with good robustness.

Fig. 8 shows the power flow of the inverter output active power, grid active power and load active power. So, part of the inverter power is used to feed the load and the rest is supplied to the grid.

Now, let's presents the simulation results corresponding to the reactive power control. The behavior of the second output $Y_{2}=I_{q}$ is shown in fig. 9. So, $I_{q}$ converges with a high precision and very fast response to its reference $I_{\text {qref }}$, which compensates the reactive power caused by the load under atmospheric condition changes and system perturbations as 
demonstrated in fig. 10. Fig. 11 shows that the reactive power injected into the grid converges to zero with negligible response time. Moreover, the unity power factor is achieved in the level of the power flow between the utility grid and the system. So, the grid current and voltage are in phase under PVG power variation, system parameters disturbance and load harmonic pollution as shown in fig. 12, fig. 13, and fig. 14 .
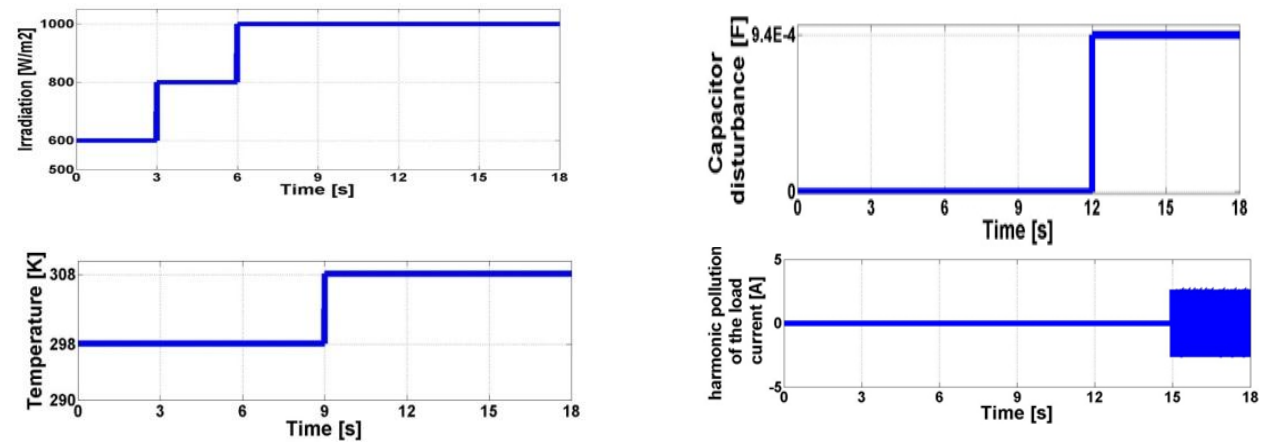

Fig. 3. Irradiation and temperature changes and disturbance values of the inverter input capacitor $C_{p}$ and harmonic pollution signal of the load current.

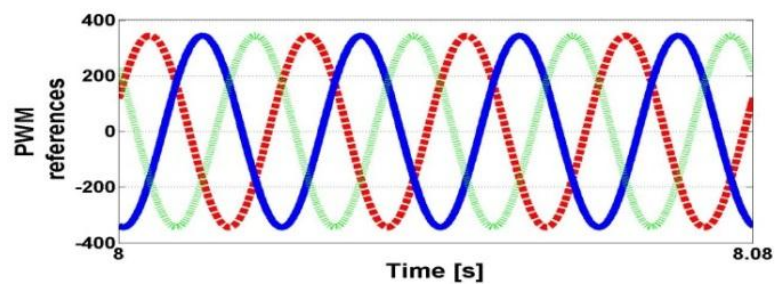

Fig. 4. The inverter three phase references output voltages.

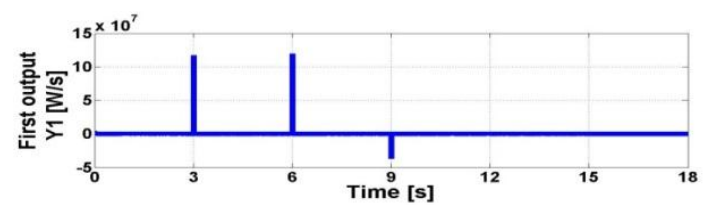

Fig. 5. The derivative of the $P V G$ power $P_{v}$ with respect to $P V G$ voltage $v_{p}$.

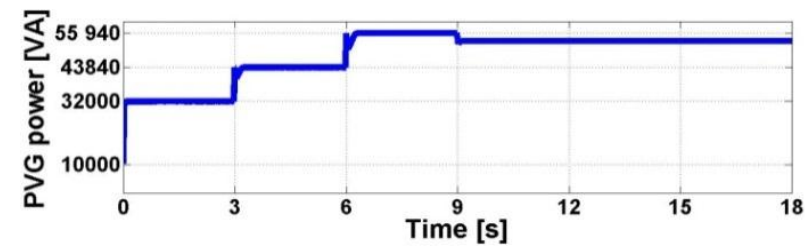

Fig. 6. MPPT achievement under abrupt variation of irradiation and temperature and in the presence of system parameter disturbance and load current harmonic pollution.

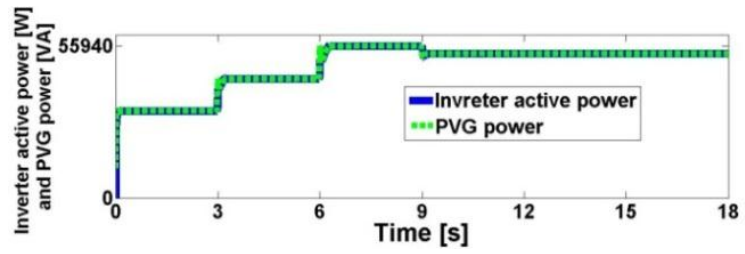

Fig. 7. The evolution of the inverter active power in function of the variation of PVG power. 


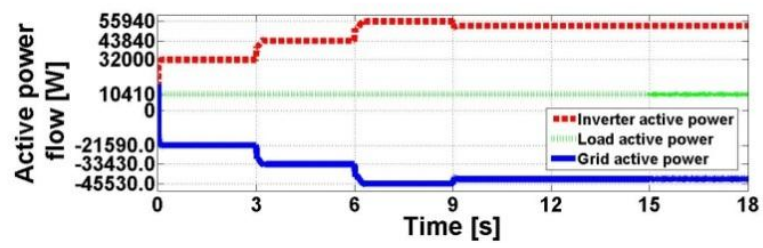

Fig. 8. Active power flow between the inverter and the grid with the active power consumed by the load.

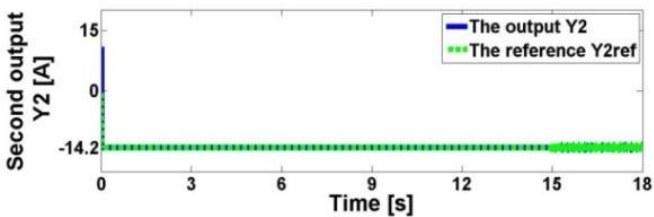

Fig. 9. The behavior of the quadratic component current of the inverter $I_{q}$.

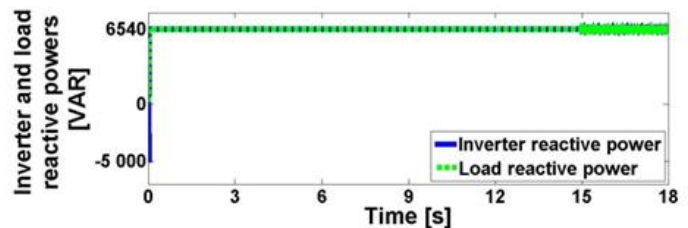

Fig. 10. Compensation of the load reactive power by the inverter output reactive power.

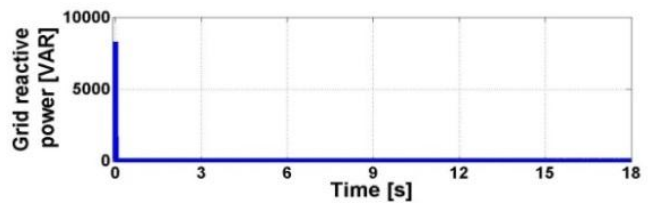

Fig. 11. Grid reactive power.

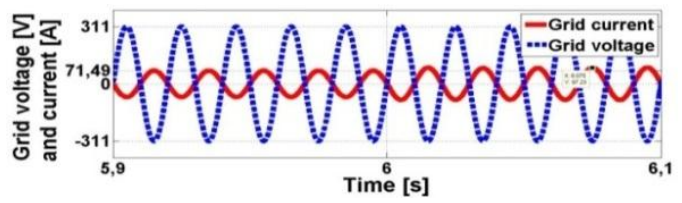

Fig. 12. Phase 'a' of the grid current and voltage (achievement of UPF under fast variation of the PVG power with a negligible response time).

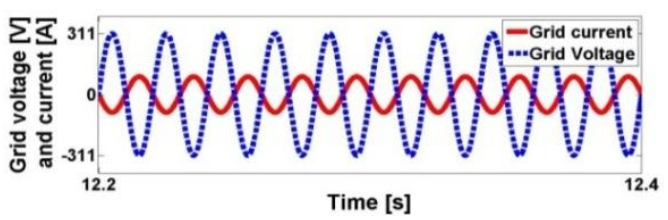

Fig. 13. Phase 'a' of the grid current and voltage (achievement of UPF in presence of inverter input capacitor disturbance).

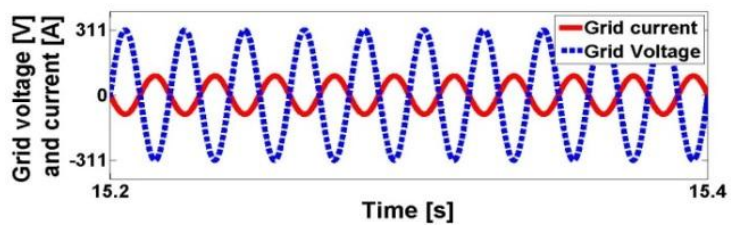

Fig. 14. Phase 'a' of the grid current and voltage (achievement of UPF during harmonic distortion of load current). 


\section{Conclusion}

This paper presented a new model and advanced control strategy of a three phase grid connected photovoltaic system with or without load. The new model has decoupled the input control, which simplifies the operation and enhances the performance of the controller. The controller has been developed by using a sliding mode control without chattering phenomena based on dq0 transformation of the new model; in order to realize the main objectives with a high performance under climatic condition changes and system disturbance. These objectives are tracking the Maximum Power Point and achieving the UPF in the level of the grid power in the presence of load or without load. Mathematical analysis has been used to demonstrate the asymptotic stability of the controlled system and simulation results proved the very good performance of the controller.

\section{References}

[1] Jinsoo Kim and Oh yang, Design of a High-Efficient Grid-Connected Three-Phase Three-Level T-Type PV Power System, International Journal of Control an Automation, Vol. 7, No. 10,pp. 65-78, 2014.

[2] J. P. Lyons and V. Vlatkovic, Power Electronics and Alternative Energy Generation, Proceedings of PESC 04, pp.16-21, Germany, June 2004.

[3] Yousry atia, M.M.Salem, Aref Eliwa and Mohamed Zahran, Simulation and Implementation of Power Control Strategy Grid Connected Photovoltaic System, WSEAS TRANSACTIONS on SYSTEMS and Control,Vol. 10, E-ISSN. 2224- 2856, 2015.

[4] M.R. Bengourina, M. Rahli and L. Hassaine, Direct power control of a grid connected photovoltaic system associated with an active power filter, Revue des Energies Renouvelables, Vol. $20 \mathrm{~N}^{\circ} 1$ pp. $99-109,2017$.

[5] Ehsan Afshari, Babak Farhangi, Frede Blaabjerg, Control Strategy for Three-Phase Grid

[6] Connected PV Inverters Enabling Current Limitation under Unbalanced Faults, IEEE Transactions on Industrial Electronics, pp. 8908 - 8918, 31 July 2017.

[7] Mateus F. Schonardie and Denizar C. Martins, Three-Phase Grid-Connected Photovoltaic System With Active And Reactive Power Control Using dq0 Transformation, pp. 1202-1207, IEEE 2008.

[8] C. Cecati, A. Dell'Aquila, M. Liserre. A novel three-phase single stage distributed power inverter. IEEE Transactions on Power Electronics, Vol. 19, Issue. 5, pp. 1226 - 1233, Sept. 2004.

[9] S. Nonaka. A novel three-phase sinusoidal PWM voltage source inverter and its application for photovoltaic power generation system, Power Conversion Conference - Nagaoka 1997, Vol. 2, pp. 755 - 758, 3-6 Aug 1997.

[10] M'hammed Guisser, Elhassane Abdelmounim, Mohammed Aboulfatah, Abdesselam ELJouni, Nonlinear Observer-Based Control for Grid Connected Photovoltaic System, IOSR Journal of Electrical and Electronics Engineering, Vol. 9, e-ISSN: 2278-1676, p-ISSN: 2320-3331, Issue. 5, PP 40-52, Sep - Oct. 2014.

[11] Evgenije Adzic, Vlado Porobic, Boris Dumnic, Nikola Celanovic, Vladimir Katic, PLL Synchrnization in Grid connected Converters, International Conference on Engineering and Technology (ICET-2013), pp. 15, Serbia May 15-17,2013.

[12] Farhan Mahmood, Luigi Vanfretti and Hossein Hooshyar, Modeling of a Detailed Photovoltaic Generation System for EMT-Type Simulation, ENERGYCON 2014, Dubrovnik, Croatia, May 13-16, 2014.

[13] M. Guisser, M. Aboulfatah, E. Abdelmounim, H. Medromi,J. Saadi. Nonlinear Control Design for Maximum Power Point Tracking and Unity Power Factor of a Grid-Connected Photovoltaic Renewable Energy Systems, IOSR Journal of Electronics and Communication Engineering, vol.9, no.5, pp.62-71, 2014. 